\title{
Research Article \\ Effects of Drugs Reabsorption Time Delays for a Pharmacokinetics Model in Humans
}

\author{
Adamu Shitu Hassan (1) and Justin M. W. Munganga \\ Department of Mathematical Sciences, College of Science, Engineering and Technology, Florida Science Campus, University of \\ South Africa, Pretoria 0003, South Africa \\ Correspondence should be addressed to Adamu Shitu Hassan; hashitu.mth@buk.edu.ng
}

Received 29 August 2021; Accepted 20 November 2021; Published 26 December 2021

Academic Editor: Chong Lin

Copyright (C) 2021 Adamu Shitu Hassan and Justin M. W. Munganga. This is an open access article distributed under the Creative Commons Attribution License, which permits unrestricted use, distribution, and reproduction in any medium, provided the original work is properly cited.

\begin{abstract}
A three-compartmental delay model is formulated to describe the pharmacokinetics of drugs subjected to both intravenous and oral doses with reabsorptions by the central compartment. Model dynamics are analyzed rigorously, and two equilibrium points are obtained to be locally asymptotically stable under certain conditions. Time delays used as lags in reabsorption of drugs by central compartment from other two compartments caused rebounds or peaks and fluctuations in the time profiles for amounts of drug in all the compartments. Sensitivity analysis revealed that elimination rates decrease the amounts in all compartments. Furthermore, reabsorption rates cause superimposition at the initial phases of the drug amount profiles; subsequently, the quantities decrease in compartment one and increase in compartments two and three, respectively.
\end{abstract}

\section{Introduction}

Pharmacokinetics (PK) is a branch of pharmacology (study of drug or medication action), derived from two Greek words pharmakon (drug) and kinetics (movement). In its simplest meaning, PK is the study of drug absorption, distribution, and elimination (related to excretion and metabolism) $[1,2]$. Closely related to PK is pharmacodynamics (PD) which refers to the relationship between drug concentration at the site of action and the resulting effect, including the time course and intensity of therapeutic and adverse effects [1]. In the concept of many authors, there is no clear distinction between PK and PD. However, according to Holford and Sheiner [3], pharmacokinetics is described as "what the body does to the drug" and pharmacodynamics as "what the drug does to the body." The most important contribution to drug research can be traced back to the pioneer work of Teorell [4] in his two famous articles which led to his title as "father of pharmacokinetics" [5]. In the articles, Toerell derived differential equations to describe the kinetics for distribution of drugs in the body and presented timeconcentration curves graphically to depicts the relation.
Administrations of drug into body system are usually carried out through two most populous routes: enteral (oral, sublingual, and rectal) and parenteral (intravenous, intraarterial injections, etc.) [6]. Entrohepatic circulation is one of the important drug transformation in the body and is a process in which administrated product excreted by liver into the bile is reabsorbed by the liver via portal blood stream [7-9]. The PK and PD of drugs after administration can be very complex as a result of various processes taking place that alter the amount of drug and/or concentrations in tissues and fluids [1]. Furthermore, measuring the amount of drug at the site of action and beyond is practically difficult if not impossible. Thus, there is a need for simplified body process representation in relation to the drug behaviour. One of the methods for body simplification process is the application of mathematical principles, using suitable compartmental models as introduced by Sheppard [10]. A compartment can represent a tissue, fluid, or group which is homogeneous and specific with respect to biological activities in the body with size determined by concentration or amount of material [11]. Based on the nature of PK of drugs in the body, compartments can be numbered and organs/ 
tissues classified according to highly perfused and less well perfused. Highly perfused compartment is also called central compartment and comprised of the blood, heart, liver, lungs, and kidney or even the whole body. The less well perfused compartment, sometimes called peripheral compartment, includes fat tissue, muscle tissue, and skeletal cerebrospinal fluid [1]. Apparently, a compartmental model provides a comprehensive form of representing body tissues/organs fluids into one, two, or multicompartments, monitors the drug's movement and behaviour, and predicts the time, amount, and concentration as it changes with time.

Generally, ordinary differential equations are among the most powerful mathematical tools used to describe models that are assumed instantaneous time transfer of material flow between and within compartments [12-15]. However, for most biological systems, this inter/intraflow of materials does not occur immediately but with time delays [16, 17]. In particular, delay may cause dynamical instabilities that generate periodic solutions, and biologically, this may affect normal physiological functions [18-21]. Many mathematical delay models of pharmacokinetics have been studied to give insight into the dynamics and pharmacodynamics of drugs. In PK models, delay can be used to represent time lag in transfer and absorption or reabsorption between one part of the body to another [7-9, 22]. For instance Steimer et al. in [8] considered two-compartmental model (body and gastrointestinal tract) with delay representing time lag for reabsorption of drug after biliary excretion from the body (first compartment) to gastrointestinal (second compartment). They found that their model exhibits qualitative agreement with the two basic experimental observations (rebounds and slow terminal kinetics) on PK of drugs undergoing enterohepatic circulation. Plusquellec and Bousquet [9] proposed another two-component model with time lag describing PK of drugs subject to enterohepatic circulation after an intravenous bolus, or an oral intake or a constant infusion. Using computer simulations, plasma level profiles that lead to rebounds and secondary peaks are obtained in accordance with experimental evidences. Labat et al. in [7] presented yet another two-component model with time delay for PK of drugs subject to enterohepatic circulation when reabsorption is repeated at unequal intervals. It was found that the first reabsorption peak area depends on time of administration and was noticeable only when a parameter was above a given threshold. In [22], analytic expression is presented for the solution of a delay differential system for any $n$ compartments with a single input and by convolution for all intakes. The result is applied to one-, two-, and three-compartmental models that provide insight into the PK of drug subjected to enterohepatic circulation among others.

From the literature presented above, it is evident that time delay play important roles in both the dynamics of PK models and physiological functions in the body. Motivated by these, in the current study, we extend threecompartmental models in $[12,23]$ by incorporating two delays, $\tau_{1}$ and $\tau_{2}$, to represent time lags for reabsorption of drug at compartment 1 (central compartment) after elimination by compartments 2 and 3, respectively. Furthermore, our new PK delay model considers intravenous and oral doses of drug at the central and peripheral compartments, respectively, in addition to elimination in all the three compartments. Most of the parameter values used are obtained from published articles optimized from experimental data to validate the model. The objectives are to find expression that can determine the amount of drug at any given time with specified delay and investigate the effects of time lag, rate constants of transfer between/out of compartments. In what follows, the paper is organized as shown. In Section 2 , the model formulation and description are given, followed by basic results in Section 3. Section 4 consists of equilibria and stability analysis, while numerical simulations and sensitivity analysis are presented in Section 5 to illustrate our equilibrium results and effects of variation of parameter values. Finally, the concluding remarks is presented in Section 6 followed by references.

\section{Model Formulation and Description}

The model consists of three compartments, with $X_{1}(t), X_{2}$ $(t)$, and $X_{3}(t)$ representing the amounts of drug at time $t$. We assume that compartment 1 (central compartment) represents highly perfused organs/tissues in the body such as the heart, liver, kidney, brain, or plasma. Compartment 2 less highly perfused organs like the gastrointestinal tract and bile while compartment 3 less perfused organs such as the adipose and skeletal muscle. The rate constants $k_{12}$ and $k_{21}$ are the kinetics constant of drug transfer between the central and compartment 2, while $k_{13}$ and $k_{31}$ are the kinetics constant of drug transfer between the central and compartment 3. There are drug eliminations (clearances) rates, $k_{10}, k_{20}$, and $k_{30}$, respectively, from each compartment. We introduce time delays $\tau_{1}$ and $\tau_{2}$, to represent the time lags in reabsorption of drugs in the central compartment from compartments 2 and 3, respectively. Thus drug leaving compartments 2 and 3 at time $t$ can be reabsorbed at central compartment at times $t+\tau_{1}$ and $t+\tau_{2}$, respectively. Drug is introduced by intravenous injection at constant rate $D_{1}$ in the central compartment, and orally into compartments 2 and 3 , respectively, at constant rates $D_{2}$ and $D_{3}$. In all the cases of transport and elimination of drugs in the compartments, we assume first-order kinetics. The schematic diagram of the model in Figure 1, together with the model equations in the following systems describe the model dynamics.

$$
\begin{aligned}
\frac{d X_{1}(t)}{d t}= & D_{1}-\left(k_{10}+k_{12}+k_{13}\right) X_{1}(t)+k_{21} X_{2}\left(t-\tau_{1}\right) \\
& +k_{31} X_{3}\left(t-\tau_{2}\right), \\
\frac{d X_{2}(t)}{d t}= & D_{2}-k_{21} X_{2}\left(t-\tau_{1}\right)-k_{20} X_{2}(t)+k_{12} X_{1}(t), \\
\frac{d X_{3}(t)}{d t}= & D_{3}-k_{31} X_{3}\left(t-\tau_{2}\right)-k_{30} X_{3}(t)+k_{13} X_{1}(t) .
\end{aligned}
$$

The model (1)-(3) satisfy the nonnegative initial data defined as 


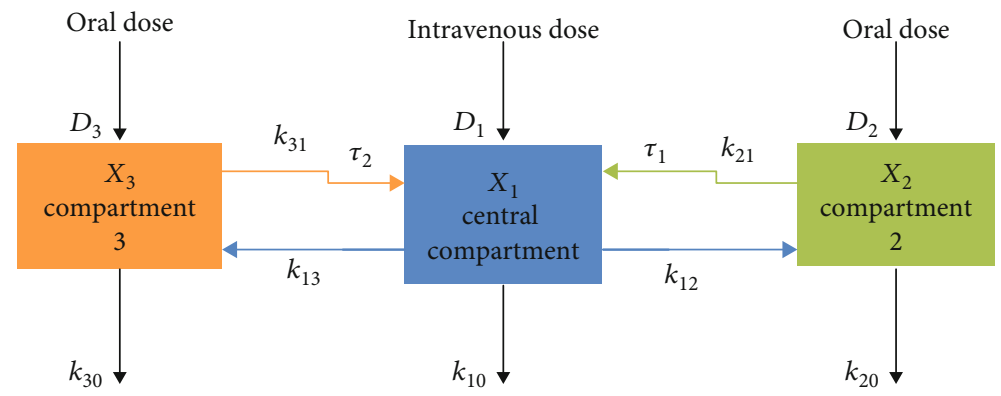

FIGURE 1: Flow diagram of the pharmacokinetics delay model with reabsorption at the central compartment.

$$
X_{1}(t)=\psi_{1}(t), X_{2}(t)=\psi_{2}(t), X_{3}(t)=\psi_{3}(t), t \in[-\tau, 0],
$$

where $\psi_{1}, \psi_{2}$, and $\psi_{3}$ belong to a Banach space of continuous functions, $\mathscr{C}$ mapping the interval $[-\tau, 0]$ into $\mathbb{R}_{+}^{3}$ $\left(\mathscr{C}\left([-\tau, 0], \mathbb{R}_{+}^{3}\right)\right)$ with $\tau=\max \left\{\tau_{1}, \tau_{2}\right\}$.

Letting $X(t)=\left[X_{1}(t) X_{2}(t) X_{3}(t)\right]^{T}$ to be vector for the amount of drug in the body at time $t$, the model systems (1), (2), and (3) can be written as

$$
\frac{d X(t)}{d t}=D+A X(t)+B_{1} X\left(t-\tau_{1}\right)+B_{2} X\left(t-\tau_{2}\right)
$$

where

$$
\begin{aligned}
D= & {\left[\begin{array}{ccc}
D_{1} & 0 & 0 \\
0 & D_{2} & 0 \\
0 & 0 & D_{3}
\end{array}\right], A=\left[\begin{array}{ccc}
-K_{1} & 0 & 0 \\
k_{12} & -k_{20} & 0 \\
k_{13} & 0 & -k_{30}
\end{array}\right], } \\
B_{1}= & {\left[\begin{array}{ccc}
0 & k_{21} & 0 \\
0 & -k_{21} & 0 \\
0 & 0 & 0
\end{array}\right], } \\
B_{2}= & {\left[\begin{array}{ccc}
0 & 0 & k_{31} \\
0 & 0 & 0 \\
0 & 0 & -k_{31}
\end{array}\right] \text { and } X(t)=\left[\begin{array}{c}
X_{1}(t) \\
X_{2}(t) \\
X_{3}(t)
\end{array}\right], } \\
& \text { where } K_{1}=k_{10}+k_{12}+k_{13} .
\end{aligned}
$$

\section{Preliminary Results}

Expressing the model equations in the form (5), it is well known from the fundamental theories of functional differential equations $[24,25]$ that model (1)-(3) admit a unique solution $\left(X_{1}(t), X_{2}(t), X_{3}(t)\right)$ with the stated initial data.

Furthermore, since the model (1)-(3) represent biological process, we assume that all parameters and variables are positive at all times $t \geq 0$.

On the boundedness of solutions, we present the following result.
Theorem 1. Assuming the positivity of solutions with initial data, the solution set $\left(X_{1}(t), X_{2}(t), X_{3}(t)\right)$ is bounded in the region

$$
\begin{aligned}
\Omega= & \left\{\left(X_{1}(t), X_{2}(t), X_{3}(t)\right) \in \mathbb{R}_{+}^{3} \mid X_{T}(t) \leq \frac{D}{K_{t}}\right\}, \\
& \text { where } K_{t}=\min \left\{k_{10}, k_{20}, k_{30}\right\}, D=D_{1}+D_{2}+D_{3} .
\end{aligned}
$$

Proof. Assuming positivity of solution with initial data and letting $X_{T}(t)=X_{1}(t)+X_{1}(t)+X_{1}(t)$ be the total amount of drug in the body at time $t$, adding the equations in models (1), (2), and (3), we have

$$
\begin{gathered}
\frac{d X_{T}(t)}{d t}=D_{1}+D_{2}+D_{3}-k_{10} X_{1}(t)-k_{20} X_{2}(t)-k_{30} X_{3}(t) \\
\leq D-K_{t} X_{T}(t) \text {, hence using Gronwall inequality } \\
X_{T}(t) \leq \frac{D}{K_{t}} \quad \text { if } \quad X_{T}(0) \leq \frac{D}{K_{t}}
\end{gathered}
$$

\section{Equilibria and Stability of the Model}

Here, we present the equilibria solutions and their stabilities for the model. At equilibrium, we have $X_{i}\left(t-\tau_{j}\right)=X_{i}(t)=$ $X_{i}^{*}$, for $i=1,2,3, j=1,2$. In the absence of intravenous and oral doses $\left(D_{1}=D_{2}=D_{3}=0\right)$, the trivial equilibrium exists and, from the model (1)-(3), is obtained to be

$$
E^{0}=\left(X_{1}^{0}, X_{2}^{0}, X_{3}^{0}\right)=(0,0,0)
$$

It is worth remarking here that the trivial equilibrium represent the initial amount of drug in the body parts when there is no intravenous and oral doses. With time, the existing amount of drug will be exhausted by the body metabolism and hence will be zero.

However, when there is introduction of either intravenous and/or oral doses $\left(D_{1} \neq D_{2} \neq D_{3} \neq 0\right)$, the nontrivial equilibrium emerged and is obtained as follows. From (1), (2), and (3), at equilibrium, we get

$$
\begin{aligned}
& D_{1}-K_{1} X_{1}^{*}+k_{21} X_{2}^{*}+k_{31} X_{3}^{*}=0, \\
& D_{2}-k_{21} X_{2}^{*}-k_{20} X_{2}^{*}+k_{12} X_{1}^{*}=0,
\end{aligned}
$$




$$
D_{3}-k_{31} X_{3}^{*}-k_{30} X_{3}^{*}+k_{13} X_{1}^{*}=0 .
$$

Let $K_{2}=k_{20}+k_{21}, K_{3}=k_{30}+k_{31}$. From (9),

$$
\begin{gathered}
X_{2}^{*}\left(k_{20}+k_{21}\right)=D_{2}+k_{12} X_{1}^{*} \text {, so that } \\
X_{2}^{*}=\frac{D_{2}+k_{12} X_{1}^{*}}{K_{2}} .
\end{gathered}
$$

Also from (13),

$$
\begin{gathered}
X_{3}^{*}\left(k_{30}+k_{31}\right)=D_{3}+k_{13} X_{1}^{*} \text {, hence } \\
X_{3}^{*}=\frac{D_{3}+k_{13} X_{1}^{*}}{K_{3}} .
\end{gathered}
$$

Substituting (15) and (17) in (11) and simplifying, gives

$$
X_{1}^{*}=\frac{D_{1}+\left(k_{21} D_{2} / K_{2}\right)+\left(k_{31} D_{3} / K_{3}\right)}{K_{1}-k_{21} k_{12} / K_{2}-k_{31} k_{13} / K_{3}} .
$$

Therefore, from Equations (15)-(18), the unique equilibrium is given by

$$
E^{*}=\left(X_{1}^{*}, X_{2}^{*}, X_{3}^{*}\right)
$$

The nontrivial equilibrium always exists when $K_{1}>$ $\left(\left(k_{21} k_{12}\right) / K_{2}+\left(k_{31} k_{13}\right) / K_{3}\right)$. It is easy to see, based on the values of $k_{i j} \mathrm{~s}$, that this condition always holds true. We now determine an arbitrary transcendental equation for the model as follows, assuming a solution of the form $X(t)=p e^{\lambda t}$, with $\lambda$ an eigenvalue and $p$ a constant vector. From (5)

$$
p e^{\lambda t} \lambda=D+A p e^{\lambda t}+B_{1} p e^{\lambda t} e^{-\lambda \tau_{1}}+B_{1} p e^{\lambda t} e^{-\lambda \tau_{2}} .
$$

Taking $p$ as an identity vector, and for nontrivial solution, we have

$$
\lambda I-D e^{-\lambda t}-A-B_{1} e^{-\lambda \tau_{1}}-B_{1} e^{-\lambda \tau_{2}}=0,
$$

where $I$ is a $3 \times 3$ identity matrix.

4.1. Stability of Trivial Equilibrium, $E^{0}=(0,0,0)$. The stability of trivial equilibrium is stated here and proved.

Theorem 2. The trivial equilibrium $E^{0}$ is locally asymptotically stable for all values of delays $\tau_{1}>0$ and $\tau_{2}>0$ whenever $D_{i}=0$, for all $i=1,2,3$.
Proof. At $E^{0}=(0,0,0), D_{1}=D_{2}=D_{3}=0$, and taking the determinant of Equation (21), we have

$$
\begin{aligned}
g(\lambda)= & (\lambda+K)\left[\left(\lambda+k_{20}+k_{21} e^{-\lambda \tau_{1}}\right)\left(\lambda+k_{30}+k_{31} e^{-\lambda \tau_{2}}\right)\right] \\
& +k_{12}\left[-k_{21} e^{-\lambda \tau_{1}}\left(\lambda+k_{30}+k_{31} e^{-\lambda \tau_{2}}\right)\right] \\
& -k_{13}\left[k_{31} e^{-\lambda \tau_{2}}\left(\lambda+k_{20}+k_{21} e^{-\lambda \tau_{2}}\right)\right]=0
\end{aligned}
$$

When $\tau_{1}=\tau_{2}=0$, Equation (22) becomes

$$
\begin{aligned}
G(\lambda)= & (\lambda+K)\left[\left(\lambda+k_{20}+k_{21}\right)\left(\lambda+k_{30}+k_{31}\right)\right] \\
& +k_{12}\left[-k_{21}\left(\lambda+k_{30}+k_{31}\right)\right]-k_{13}\left[k_{31}\left(\lambda+k_{20}+k_{21}\right)\right] .
\end{aligned}
$$

It can be seen that

$$
\begin{aligned}
G(0)= & K\left[\left(k_{20}+k_{21}\right)\left(k_{30}+k_{31}\right)\right]-k_{12}\left[k_{21}\left(k_{30}+k_{31}\right)\right] \\
& -k_{13}\left[k_{31}\left(k_{20}+k_{21}\right)\right], \\
= & K K_{2} K_{3}-k_{12} k_{21} K_{3}-k_{13} k_{31} K_{2}=k_{10} K_{2} K_{3}+k_{12} k_{20} K_{3} \\
& +k_{13} k_{30} K_{2}>0, \text { and } \lim _{\lambda \longrightarrow \infty} G(\lambda)=+\infty .
\end{aligned}
$$

This implies that all the eigenvalues of $G(\lambda)=0$ have negative real parts; hence, $E^{0}$ is stable for $\tau_{1}=\tau_{2}=0$.

When $\tau_{1}>0, \tau_{2}>0$, from (22), after simplification, we again have

$$
\begin{gathered}
G(0)=k_{10} K_{2} K_{3}+k_{12} k_{20} K_{3}+k_{13} k_{30} K_{2}>0, \text { and } \\
\lim _{\lambda \longrightarrow \infty} G(\lambda)=+\infty .
\end{gathered}
$$

Hence, $G(\lambda)=0$ has no positive real roots. Therefore, $E^{0}$ is absolutely stable for all delays $\tau_{1}>0$ and $\tau_{2}>0$.

4.2. Stability of Nontrivial Equilibrium, $E^{*}=\left(X_{1}^{*}, X_{2}^{*}, X_{3}^{*}\right)$. The equilibrium $E^{*}$ emerged whenever either or all of $D_{i}>$ 0 , for $i=1,2,3$. With this condition, the determinant of transcendental Equation (21) is given as

$$
\begin{aligned}
H(\lambda)= & \left(\lambda-D_{1} e^{-\lambda t}+K_{1}\right)\left[\left(\lambda-D_{2} e^{-\lambda t}+k_{20}+k_{21} e^{-\lambda \tau_{1}}\right)\right. \\
& \left.\cdot\left(\lambda-D_{3} e^{-\lambda t}+k_{30}+k_{31} e^{-\lambda \tau_{2}}\right)\right] \\
& -k_{12} k_{21} e^{-\lambda \tau_{1}}\left(\lambda-D_{3} e^{-\lambda t}+k_{30}+k_{31} e^{-\lambda \tau_{2}}\right) \\
& -k_{13}\left[k_{31} e^{-\lambda \tau_{2}}\left(\lambda-D_{2} e^{-\lambda t}+k_{20}+k_{21} e^{-\lambda \tau_{1}}\right)\right]=0 .
\end{aligned}
$$

Hence, Equation (26) can be express as

$$
H(\lambda)=P(\lambda)+Q(\lambda)
$$


where $P$ and $Q$ are polynomials of degrees 3 and 2, respectively, defined as follows:

$$
\begin{gathered}
P(\lambda)=\lambda^{3}+p_{2} \lambda^{2}+p_{1} \lambda+p_{0}, \\
Q(\lambda)=q_{2} \lambda^{2}+q_{1} \lambda+q_{0}, \text { with } \\
p_{2}=D_{3} e^{-\lambda t}-k_{30}+D_{2} e^{-\lambda t}-k_{20}+D_{1} e^{-\lambda t}+K_{1}, \\
p_{1}=\left(-D_{2} e^{-\lambda t}+k_{20}\right)\left(-D_{3} e^{-\lambda t}+k_{30}\right) \\
-\left(-D_{1} e^{-\lambda t}+K\right)\left(-D_{3} e^{-\lambda t}+k_{30}-D_{2} e^{-\lambda t}+k_{20}\right) \\
p_{0}=\left(-D_{1} e^{-\lambda t}+K_{1}\right)\left(-D_{3} e^{-\lambda t}+k_{30}\right)\left(-D_{2} e^{-\lambda t}+k_{20}\right) \\
q_{1}=k_{31} e^{-\lambda \tau_{2}}\left(-D_{2} e^{-\lambda t}+k_{20}\right)+k_{21} e^{-\lambda \tau_{1}}\left(-D_{3} e^{-\lambda t}+k_{30}\right) \\
+k_{21} k_{31} e^{-\lambda \tau_{1}} e^{-\lambda \tau_{2}}-\left(-D_{1} e^{-\lambda t}+K_{1}\right)\left(k_{21} e^{-\lambda \tau_{1}}+k_{31} e^{-\lambda \tau_{2}}\right) \\
-k_{12} k_{21} e^{-\lambda \tau_{1}}-k_{13} k_{31} e^{-\lambda \tau_{2}}, \\
+\left(-D_{1} e^{-\lambda t}+K_{1}\right) k_{21} k_{31} e^{-\lambda \tau_{1}} e^{-\lambda \tau_{2}} \\
q_{0}=\left(-D_{1} e^{-\lambda t}+K_{1}\right)\left(D_{2} e^{-\lambda t}-k_{20}\right) k_{31} e^{-\lambda \tau_{2}} \\
+\left(-D_{1} e^{-\lambda t}+K_{1}\right)\left(-D_{3} e^{-\lambda t}+k_{30}\right) k_{21} e^{-\lambda \tau_{1}} \\
\left.+D_{2} e^{-\lambda t}-k_{20}+k_{21} e^{-\lambda \tau_{1}}\right)
\end{gathered}
$$

To discuss the stability of $E^{*}$, we first study the distribution of roots for Equation (26). It can be seen from Equation (27) that

$$
\begin{aligned}
H(0)= & -\left(D_{1} D_{2} D_{3}+D_{1} K_{2} K_{3}+K_{1} K_{3} D_{2}+K_{1} K_{2} D_{3}\right. \\
& \left.+k_{12} k_{21} k_{30}+k_{12} k_{21} k_{31}+k_{13} k_{31} k_{20}+k_{13} k_{31} k_{21}\right) \\
& +\left(D_{1} D_{2} K_{3}+D_{1} D_{3} K_{2}+k_{12} k_{21} D_{3}+k_{13} k_{31} D_{2}\right. \\
& \left.+K_{1} D_{2} D_{3}+K_{1} K_{2} K_{3}\right)<0, \text { and } \\
& \lim _{\lambda \longrightarrow \infty} H(\lambda)=+\infty
\end{aligned}
$$

This indicates that Equation (27) has at least one positive root in $[0,+\infty)$. However, when both $\tau_{1}>0$ and $\tau_{2}>0$, Equation (26) will be too complicated for analysis and any meaningful conclusion; thus, for further discussions, we will consider one case $\left(\tau_{1}>0, \tau_{2} \in\left[0, \tau_{20}^{*}\right)\right)$, where $\left[0, \tau_{20}^{*}\right)$ is a stable interval for $\tau_{2}$, using the approaches in $[20,26]$. However, for the remaining two cases: case $2, \tau_{1}=\tau_{2}=\tau$, and case $3, \tau_{2}>0, \tau_{1} \in\left[0, \tau_{10}^{*}\right)$, where $\left[0, \tau_{20}^{*}\right)$ is a stable interval for $\tau_{1}$, we will use numerical simulations to illustrate

Case 1: $\tau_{1}>0, \tau_{2} \in\left[0, \tau_{20}^{*}\right)$.

In this case, we consider Equation (26) with $\tau_{2}$ in its stable interval and $\tau_{1}$ as the bifurcation parameter. Equation (26) will now be expressed as

$$
H(\lambda)=P(\lambda)+Q_{1}(\lambda) e^{-\lambda \tau_{1}}+Q_{2}(\lambda) e^{-\lambda \tau_{2}},
$$

where $Q_{1}$ and $Q_{2}$ are defined as

$$
\begin{gathered}
Q_{1}(\lambda)=-k_{21} \lambda^{2}+\left(q_{3}+q_{4}\right) \lambda+q_{5}+q_{6}, \\
Q_{2}(\lambda)=-k_{31} \lambda^{2}+q_{7} \lambda+q_{8}, \text { with } \\
q_{3}=k_{21}\left[\left(-D_{3}+k_{30}\right)-\left(-D_{1}+K_{1}\right)-k_{12}\right], \\
q_{4}=q_{41} e^{-\lambda \tau_{2}}, q_{41}=k_{21} k_{31}, \\
q_{5}=\left(-D_{1}+K_{1}\right)\left(-D_{3}+k_{30}\right) k_{21}, q_{6}=q_{61} e^{-\lambda \tau_{2}}, \\
q_{61}=\left(-D_{1}+K_{1}\right) k_{21} k_{31}-k_{13} k_{31} k_{21}, \\
q_{7}=k_{31}\left(-D_{2}+k_{20}\right)-\left(-D_{1}+K_{1}\right) k_{31}-k_{13} k_{31}, \\
q_{8}=\left(-D_{1}+K_{1}\right)\left(D_{2}-k_{20}\right) k_{31}+\left(-k_{13} k_{31}\left(-D_{2}+k_{20}\right)\right) .
\end{gathered}
$$

Assuming that $\lambda=i y, y \in \mathbb{R}_{+}$is the root of (30). Substituting, simplifying, and separating the real and imaginary parts, we obtain

$$
\begin{gathered}
P_{11} \cos y_{1} \tau_{1}+Q_{11} \sin y_{1} \tau_{1}=N_{11}, \\
Q_{11} \cos y_{1} \tau_{1}+P_{11} \sin y_{1} \tau_{1}=M_{11}, \text { where } \\
P_{11}=y_{1}^{2} k_{21}+y_{1} \sin y_{1} \tau_{2}+q_{5}+q_{61} \cos y_{1} \tau_{2}, Q_{11}=y_{1}^{2} q_{3} \\
+y_{1} q_{4} \cos y_{1} \tau_{2}-q_{61} \sin y_{1} \tau_{2},
\end{gathered}
$$

$$
M_{11}=y_{1}^{3}-y_{1} p_{1}+\left(y_{1}^{2} k_{31}+q_{8}\right) \sin y_{1} \tau_{2}-q_{7} y_{1} \cos y_{1} \tau_{2},
$$

$N_{11}=y_{1}^{2} p_{2}+p_{0}+\left(y_{1}^{2} k_{31}+q_{8}\right) \cos y_{1} \tau_{2}+q_{7} y_{1} \sin y_{1} \tau_{2}$.

To get an expression for $y_{1}$, we square both sides of (32) and (33) and add, so that

$$
\begin{gathered}
y_{1}^{6}+y_{1}^{4}\left(k_{21}^{2}+p_{2}^{2}\right)+y_{1}^{2}\left[\left(q_{3}+q_{4} \cos y_{1} \tau_{2}\right)^{2}\right] \\
+\sum_{i=1}^{4} f_{i}\left(y_{1}\right)=0, \text { where } \\
f_{1}=2 y_{1}^{2} k_{21}\left[y_{1} \sin y_{1} \tau_{2}+\left(q_{5}+q_{61} \cos y_{1} \tau_{2}\right)\right] \\
+\left(q_{5}+q_{61} \cos y_{1} \tau_{2}\right)^{2} \\
f_{2}=-2 y_{1}\left(q_{3}+q_{4} \cos y_{1} \tau_{2}\right) q_{61} \sin y_{1} \tau_{2}+\left(q_{61} \sin y_{1} \tau_{2}\right)^{2} \\
f_{3}=-2 y_{1}^{3}\left[-y_{1} p_{1}+\left(y_{1}^{2} k_{31}+q_{8}\right) \sin y_{1} \tau_{2}-y_{1} q_{7} \cos y_{1} \tau_{2}\right] \\
+\left[-y_{1} p_{1}+\left(y_{1}^{2} k_{31}+q_{8}\right) \sin y_{1} \tau_{2}-y_{1} q_{7} \cos y_{1} \tau_{2}\right]^{2}
\end{gathered}
$$


TABLe 1: Baseline values for the parameters of model (1)-(3).

\begin{tabular}{|c|c|c|c|}
\hline Parameter & Description & Value & Reference \\
\hline$\overline{D_{1}}$ & Rate of intravenous dose to $X_{1}$ & $50 \mathrm{mg} \mathrm{min}^{-1}$ & [25] \\
\hline$D_{2}$ & Rate of oral dose to $X_{2}$ & $40 \mathrm{mg} \mathrm{min}^{-1}$ & [26] \\
\hline$D_{3}$ & Rate of oral dose to $X_{3}$ & $40 \mathrm{mg} \mathrm{min}^{-1}$ & Assumed \\
\hline$k_{10}$ & Rate of central body elimination of drug & $0.172 \mathrm{~min}^{-1}$ & [2] \\
\hline$k_{12}$ & Rate of transporting drug from central to compartment 2 & $0.373 \mathrm{~min}^{-1}$ & [2] \\
\hline$k_{13}$ & Rate of transporting drug from central to compartment 3 & $0.0367 \mathrm{~min}^{-1}$ & [2] \\
\hline$k_{21}$ & Reabsorption rate of drug from compartment 1 to central & $0.103 \mathrm{~min}^{-1}$ & [2] \\
\hline$k_{20}$ & Rate of drug elimination from compartment 2 & $0.00828 \mathrm{~min}^{-1}$ & [2] \\
\hline$k_{30}$ & Rate of drug elimination from compartment 3 & $0.00784 \mathrm{~min}^{-1}$ & [2] \\
\hline$k_{31}$ & Reabsorption rate of drug from compartment 3 to central & $0.0124 \mathrm{~min}^{-1}$ & [2] \\
\hline$\tau_{1}$ & Time lag for reabsorption of drug from compartment 2 to 1 & {$[1,50] \min$} & [11] \\
\hline$\tau_{2}$ & Time lag for reabsorption of drug from compartment 3 to 1 & {$[1,90] \min$} & [11] \\
\hline
\end{tabular}

$$
\begin{aligned}
f_{4}= & 2 y_{1}^{2} p_{2}\left[y_{1}\left(k_{31} y_{1}+q_{7} \sin y_{1} \tau_{2}\right)+\left(p_{0}+q_{8} \cos y_{1} \tau_{2}\right)\right] \\
& +\left[y_{1}\left(k_{31} y_{1}+q_{7} \sin y_{1} \tau_{2}\right)+\left(p_{0}+q_{8} \cos y_{1} \tau_{2}\right)\right]^{2}
\end{aligned}
$$

Without loss of generality, we assume that Equation (37) has three positive roots denoted as $y_{11}, y_{12}$, and $y_{13}$. For any given root, the critical delay is therefore given by

$$
\begin{gathered}
\tau_{1 j}^{m}=\frac{1}{y_{1 j}} \cos ^{-1}\left(\frac{Q_{11} M_{11}+P_{11} N_{11}}{P_{11}^{2}+Q_{11}^{2}}\right)+\frac{2 \pi m}{y_{1 j}}, \\
j=1,2,3, m=0,1,2, . \cdots
\end{gathered}
$$

Therefore, when $\tau_{1}=\tau_{1 j}^{m}$, for $j=1,2,3, m=0,1,2, \cdots$, then $\pm i y_{1 j}$ is a pair of purely imaginary roots of Equation (30) for $\tau_{2} \in\left(0, \tau_{20}^{*}\right)$. It is easy to see that the sequence $\left\{\tau_{1 j}^{m}\right\}$ is monotone increasing so that $\lim _{m \longrightarrow \infty} \tau_{1 j}^{m}=\infty$.

Let define

$$
\tau_{10}^{*}=\min _{1 \leq j \leq 3, m \geq 0}\left\{\tau_{1 j}^{m}\right\}
$$

In order to be sure that $\pm i y_{1 j}$ are simple purely imaginary roots of Equation (30), we proceed by differentiating (21) with respect to $\tau_{1}$ to get

$$
\begin{aligned}
{\left[\frac{d \lambda}{d \tau_{1}}\right]^{-1}=} & \frac{3 \lambda^{2}+2 p_{2} \lambda+p_{1}+e^{-\lambda \tau_{1}}\left[-2 k_{21} \lambda+q_{3}+q_{41} e^{-\lambda \tau_{2}}-\tau_{2} e^{-\lambda \tau_{2}}\left(q_{41}+q_{61}\right)\right]}{\lambda e^{-\lambda \tau_{1}}\left[-k_{21} \lambda^{2}+\left(q_{3}+q_{41} e^{-\lambda \tau_{2}}\right) \lambda+q_{5}+q_{61} e^{-\lambda \tau_{2}}\right]} \\
& +\frac{e^{-\lambda \tau_{2}}\left[\left(-2 \lambda k_{31}+q_{7}\right)-\tau_{2}\left(-\lambda^{2} k_{31}+\lambda q_{7}+q_{8}\right)\right]}{\lambda e^{-\lambda \tau_{1}}\left[-k_{21} \lambda^{2}+\left(q_{3}+q_{41} e^{-\lambda \tau_{2}}\right) \lambda+q_{5}+q_{61} e^{-\lambda \tau_{2}}\right]}-\frac{\tau_{1}}{\lambda} .
\end{aligned}
$$

Substituting $\lambda=i y_{1}$ and simplifying, we have

$$
\begin{aligned}
{\left[\frac{d \lambda}{d \tau_{1}}\right]^{-1}=} & \frac{3 \lambda^{2}+2 p_{2} \lambda+p_{1}+P_{11} \cos y_{1} \tau_{1}+P_{12} \sin y_{1} \tau_{1}+\left[2 y_{1} p_{2}+P_{12} \cos y_{1} \tau_{1}-P_{11} \sin y_{1} \tau_{1}\right] i}{R_{11} \cos y_{1} \tau_{1}+R_{12} \sin y_{1} \tau_{1}+\left[R_{12} \cos y_{1} \tau_{1}-R_{11} \sin y_{1} \tau_{1}\right] i} \\
& +\frac{Q_{11} \cos y_{1} \tau_{2}+Q_{12} \sin y_{1} \tau_{2}+\left[Q_{12} \cos y_{1} \tau_{2}-Q_{11} \sin y_{1} \tau_{2}\right] i}{R_{11} \cos y_{1} \tau_{1}+R_{12} \sin y_{1} \tau_{1}+\left[R_{12} \cos y_{1} \tau_{1}-R_{11} \sin y_{1} \tau_{1}\right] i} \frac{\tau_{1}}{i y_{1}}, \text { where } \\
P_{21}= & q_{3}+q_{41} \cos y_{1} \tau_{2}-\tau_{2} q_{41} \cos y_{1} \tau_{2}-\tau_{2} q_{61} \cos y_{1} \tau_{2},
\end{aligned}
$$

$$
\begin{gathered}
P_{22}=-2 y k_{21}-q_{41} \sin y_{1} \tau_{2}+\tau_{2} q_{41} \sin y_{1} \tau_{2}+\tau_{2} q_{61} \sin y_{1} \tau_{2}, \\
Q_{21}=q_{7}-\tau_{2} y^{2} k_{31}-\tau_{2} q_{8}, Q_{22}=-2 y k_{31}-\tau_{2} y q_{7}, \\
R_{21}=y^{2} q_{3}+y^{2} q_{41} \cos y_{1} \tau_{2}, R_{22}=y^{3} k_{21}+y^{2} q_{41} \sin y_{1} \tau_{2} .
\end{gathered}
$$

Hence

$$
\begin{gathered}
{\left[\frac{d(\operatorname{Re}(\lambda))}{d \tau_{1}}\right]_{\tau_{1}=\tau_{10}^{*}}^{-1}=\operatorname{Re}\left[\frac{d \lambda}{d \tau_{1}}\right]_{\tau_{1}=\tau_{10}^{*}}^{-1}} \\
=\frac{P_{3 R}\left(P_{1 R}+P_{2 R}\right)+Q_{3 I}\left(Q_{1 I}+Q_{2 I}\right)}{P_{3 R}^{2}+Q_{3 I}^{2}}, \text { where } \\
P_{1 R}=-3 y_{1}^{*}+p_{1}+P_{21} \cos y_{1}^{*} \tau_{1}^{*}+P_{22} \sin y_{1}^{*} \tau_{1}^{*}, P_{2 R} \\
=Q_{11} \cos y_{1}^{*} \tau_{2}+Q_{22} \sin y_{1}^{*} \tau_{2}, \\
P_{3 R}=R_{21} \cos y_{1}^{*} \tau_{1}^{*}+R_{22} \sin y_{1}^{*} \tau_{1}^{*}, Q_{1 I}=2 y_{1}^{*} p_{2} \\
+P_{22} \cos y_{1}^{*} \tau_{1}^{*}-P_{21} \sin y_{1}^{*} \tau_{1}^{*}, \\
Q_{2 I}=Q_{22} \cos y_{1}^{*} \tau_{2}-Q_{21} \sin y_{1}^{*} \tau_{2} .
\end{gathered}
$$

Clearly, when $P_{3 R}\left(P_{1 R}+P_{2 R}\right)+Q_{3 I}\left(Q_{1 I}+Q_{2 I}\right) \neq 0$ is satisfied, it follows that $\operatorname{Re}\left[d \lambda / d \tau_{1}\right]_{\tau_{1}=\tau_{10}^{*}}^{-1} \neq 0$.

According to the Hopf bifurcation theorem as stated in [26], this result can be summarized below.

Theorem 3. Suppose Equation (30) admits at least one positive root and $\tau_{2}$ is in its stable interval $\left[0, \tau_{20}^{*}\right)$. The nontrivial equilibrium $E^{*}$ is locally asymptotically stable for any $\tau_{1} \in[$ $\left.0, \tau_{10}^{*}\right)$. Furthermore, the model (1)-(3) will undergo the Hopf bifurcation at $E^{*}$ when $\tau_{1}=\tau_{10}^{*}$ and family of periodic solutions bifurcate from the equilibrium when $\tau_{1}>\tau_{10}^{*}$. 

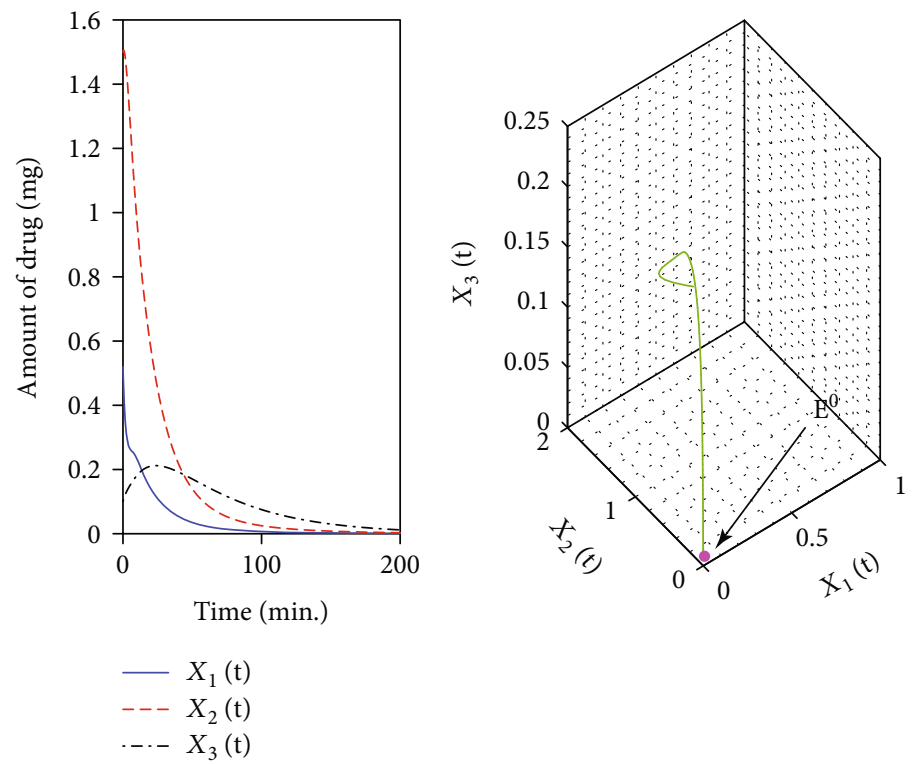

(a)
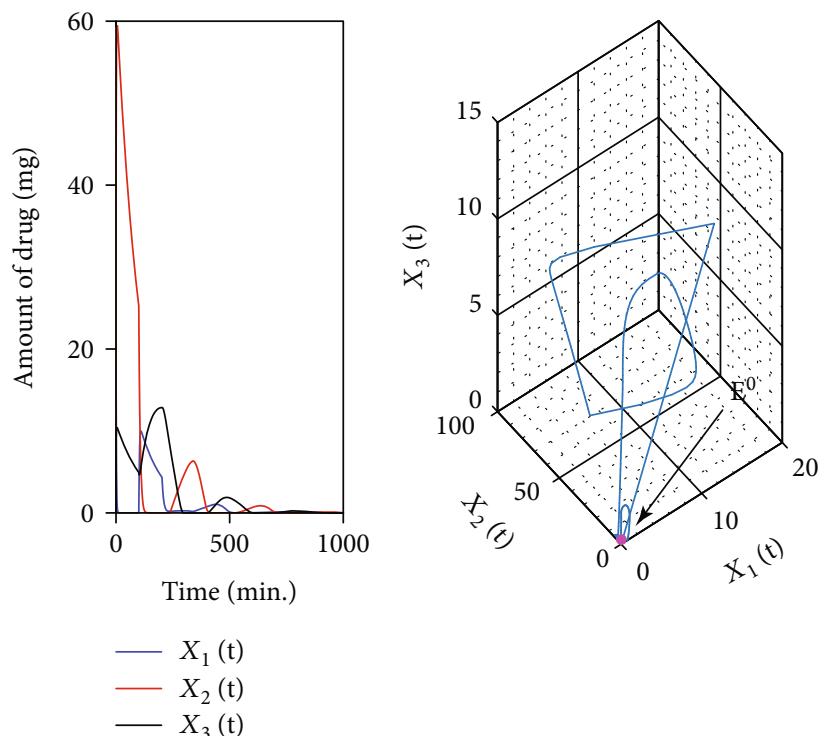

(b)

FIgURE 2: Numerical simulations displaying the local stability of trivial equilibrium $E^{0}$, using parameter values in Table 1 , using (a) small delays $\left(\tau_{1}=5, \tau_{2}=4\right)$ and (b) large delays $\left(\tau_{1}=100, \tau_{2}=100\right)$.

\section{Numerical Simulations}

In this section, we carry out some numerical experiments using parameter values as displayed in Table 1, obtained from experimental data in the literature to illustrate our theoretical results. In addition, we show the phenomenon of the Hopf bifurcation and sensitivity analysis of key parameters to illustrate effects of time delay for reabsorption of drugs, and eliminations in the model systems (1), (2), and (3).

Figure 2 illustrates the local asymptotic stability of trivial equilibrium $E^{0}$ using different delay values as proved in Theorem 2. It can be observed in both Figures 2(a) and 2(b) that when there is no intravenous and oral doses, irrespective of the delay values $\left(\tau_{1}=5\right.$ or 100 and $\tau_{2}=4$ or 100$)$, the amount of drug in the compartments will be exhausted (tends to 0 ) as time progresses.

5.1. Numerics for Stability of Equilibria. The dynamics of case 1 , where $\tau_{1}>0$ and $\tau_{2} \in\left[0, \tau_{20}^{*}\right)$ is illustrated in Figure 3 using time series and phase portrait graphs. In Figure $3(\mathrm{a})$, the local asymptotic stability of $E^{*}$ is shown when $\tau_{1}=35<\tau_{10}^{*}=44.50, \tau_{2}=20$. However, when $\tau_{1}=$ $45.60>\tau_{10}^{*}=44.50, \tau_{2}=20, E^{*}$ loses its stability as can be seen in Figure 3(a), and family of periodic solutions bifurcates from the equilibrium point $E^{*}$. 

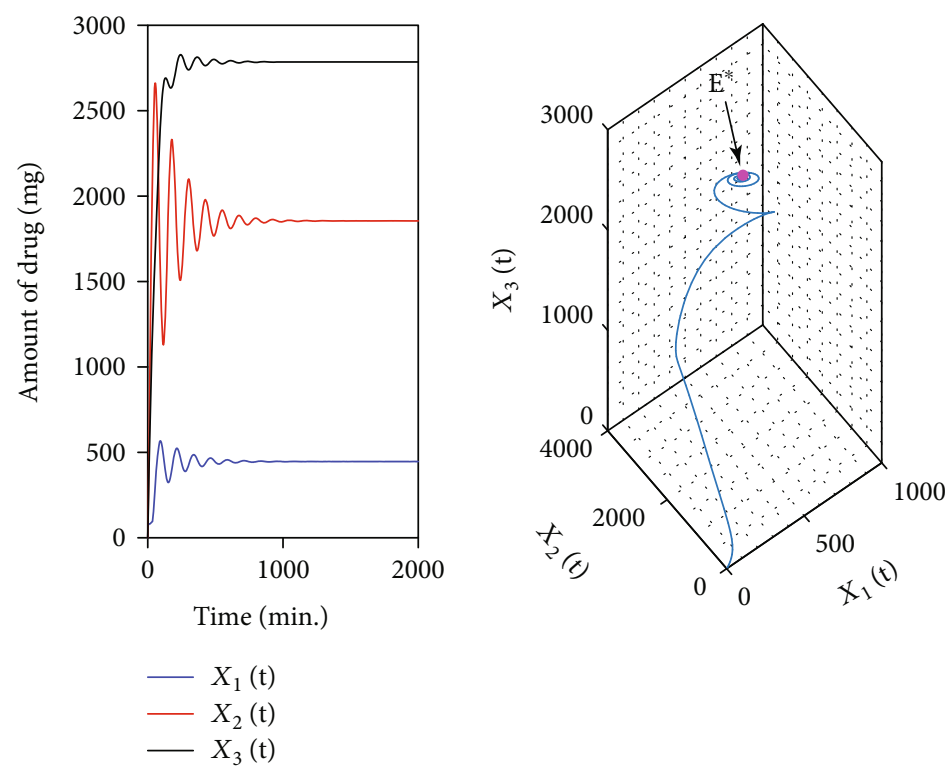

(a)
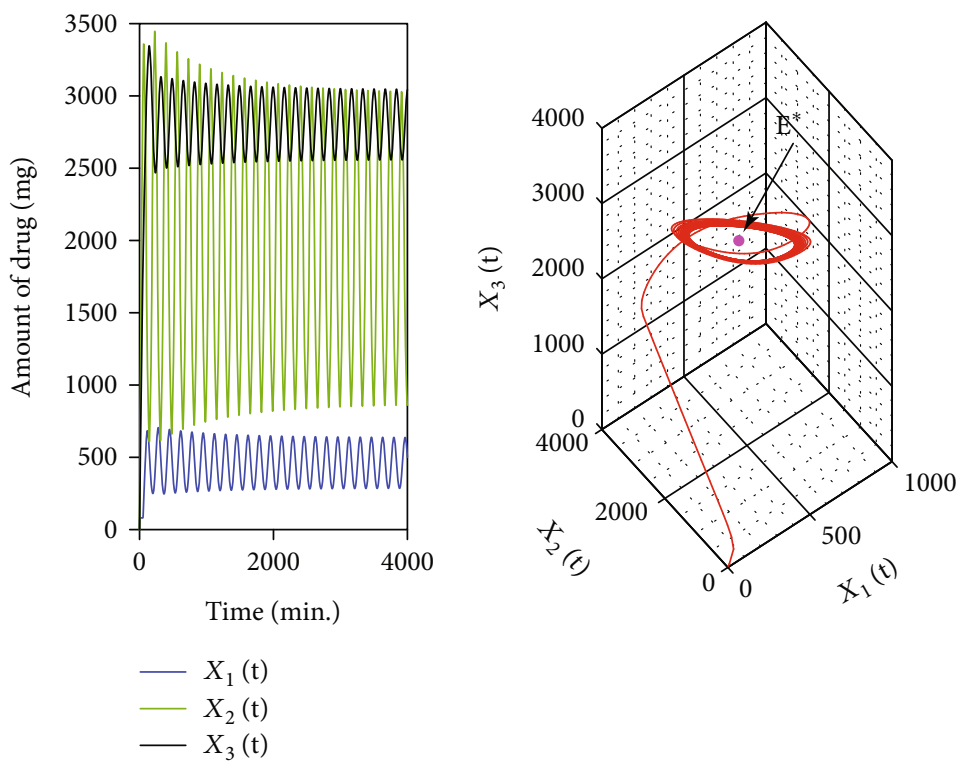

(b)

FIgURE 3: Numerical simulations for the dynamics of nontrivial equilibrium $E^{*}$ showing case 1 with time series and phase portrait graphs, in (a) local stability when $\tau_{1}=35<\tau_{10}^{*}=44.50, \tau_{2}=15$ and (b) instability for $\tau_{1}=45.60>\tau_{10}^{*}=44.50, \tau_{2}=20$, showing the Hopf bifurcation, using parameter values in Table 1 .

In Figure 4(a), the local stability of $E^{*}$ is depicted when case 2 is considered. When $\tau_{2}=29<\tau_{20}^{*}=33.23, \tau_{1}=40 \in$ [ $\left.0, \tau_{10}^{*}\right)$, the nontrivial equilibrium $E^{*}$, is shown to be locally asymptotically stable. The equilibrium then undergoes the Hopf bifurcation at $\tau_{2}=\tau_{20}^{*}=33.23$; thereafter, $E^{*}$ becomes unstable and family of solutions bifurcates from it as evident from Figure 4(b).

Similarly, case 3 is depicted in Figure 5. Here, the local asymptotic stability of the equilibrium $E^{*}$ is shown when in Figure 5(a), where $\tau_{1}=\tau_{2}=25<\tau_{c}^{*}=42.35$ are considered. However, when $\tau=44.35>\tau_{c}^{*}$, $E^{*}$ loses its stability resulting in family of periodic solutions bifurcating from the equilibrium as displayed in Figure 5(b).

Remark 4. It is worth mentioning here that the practical implication of the stability for nontrivial equilibrium $E^{*}$ is that the amount of drugs will remain fixed at any given time in the three body parts considered whenever the reabsorption time from compartments 2 and 3 to 1 does not exceed certain critical value. However, when the absorption time exceeds such time, then the amounts of drug in the three body parts will be fluctuating. 

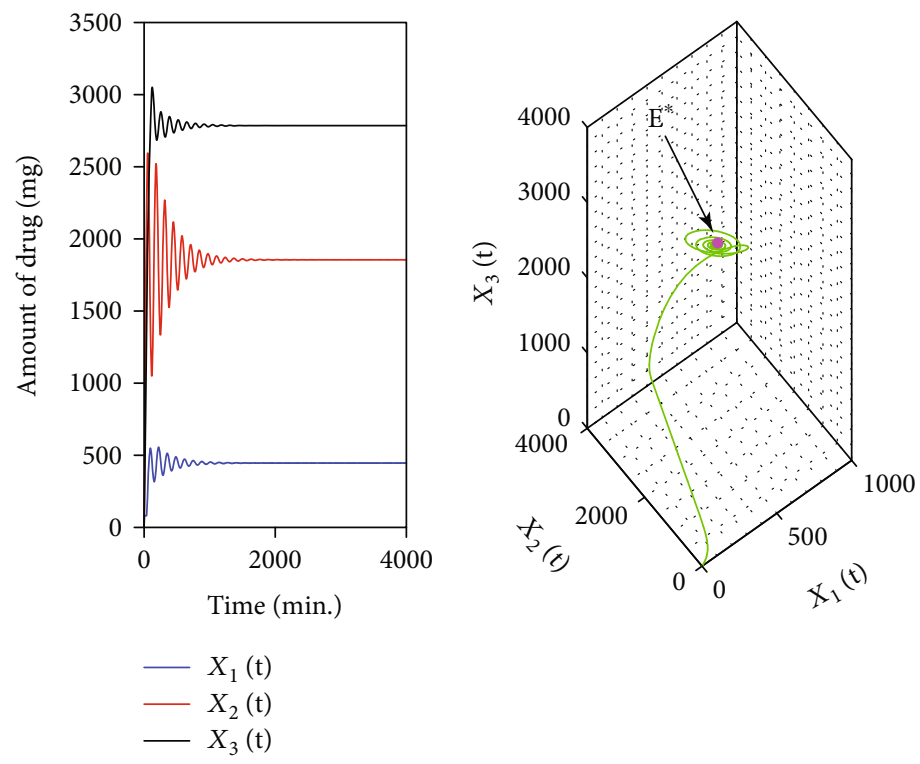

(a)
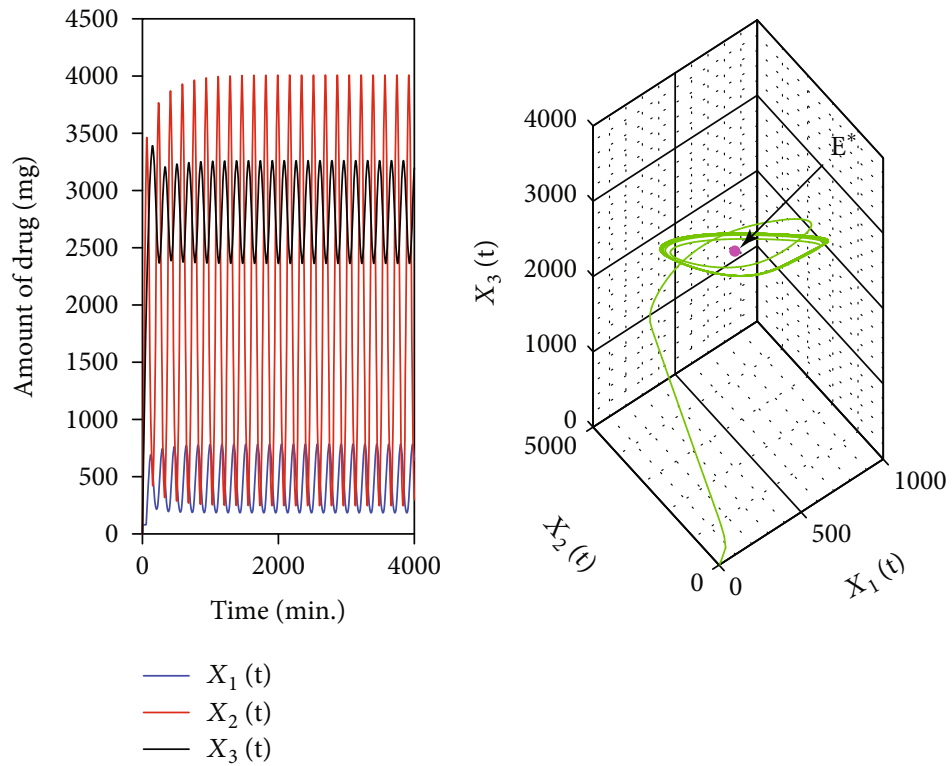

(b)

FIgURE 4: Numerical simulations for the local stability of trivial equilibrium $E^{*}$, displaying case 2, using parameter values in Table 1: (a) $\tau_{1}=40, \tau_{2}=29$, (b) $\tau_{1}=43, \tau_{2}=34.80$.

5.2. Sensitivity Analysis of Parameters. In this section, we will show the variations of drug amounts as certain parameter values in the model (1)-(3) are varied using repeated computer simulations, so as to compare with other models in the literature.

We start with effect of time lag in reabsorption of drugs by compartment 1 (central compartment) from compartments 2 and 3. In Figures 6(a) and 6(b), we varied the values of $\tau_{1}=12,27,40$ with negligible $\tau_{2}=0.1$ in order to see the behaviour of drug's amount as time progresses. It can be observed that as $\tau_{1}$ is increased, there are increase in number of rebounds or peaks in the time profiles of $X_{1}$ and $X_{2}$ in central and compartments 2, respectively. Similarly, when value $\tau_{2}$ is varied, as $\tau_{2}=30,60,90$, with negligible $\tau_{1}=0.1$, it can be seen from Figures 6(c) and 6(d) that the number of rebounds increased in the graphs of central and compartments 3 as time progresses. The implication of time lag on pharmacokinetics profile as reported in [8] is that the three phases can be distinguished. In the initial distribution phase, the amount of drug increases in the body with increase in time and delay, and the terminal distribution phase is where the amount stabilizes. Between these two phases, there is the third phase and transient phase, where the effect of delay is apparent with presence of rebounds or peaks. Therefore, as 

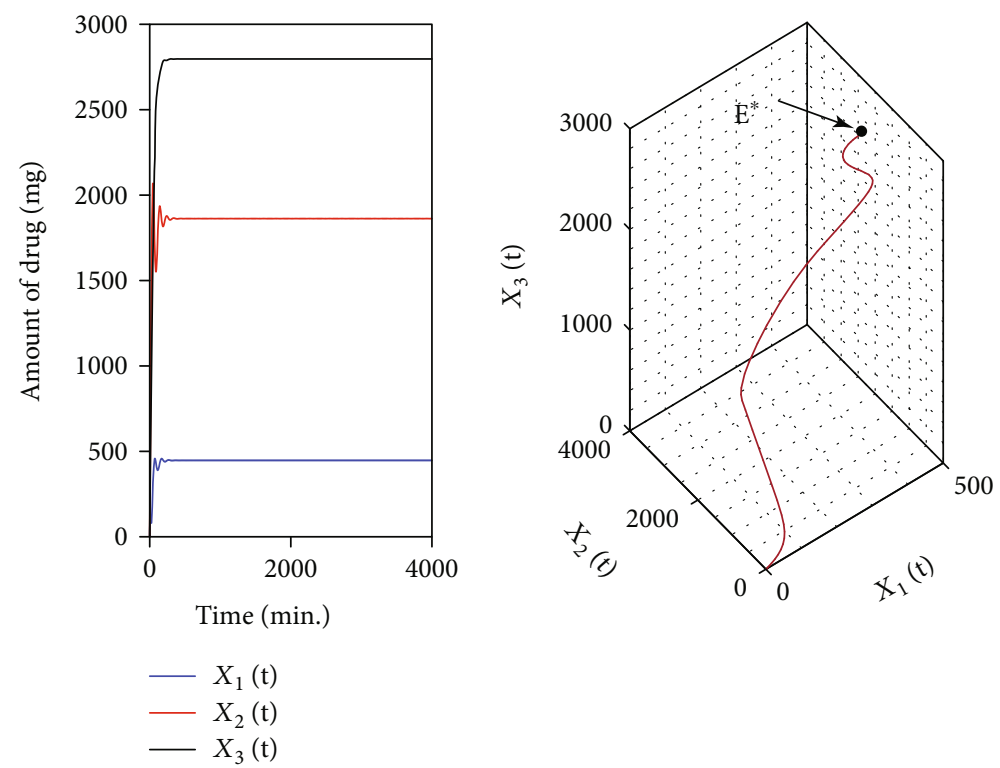

(a)
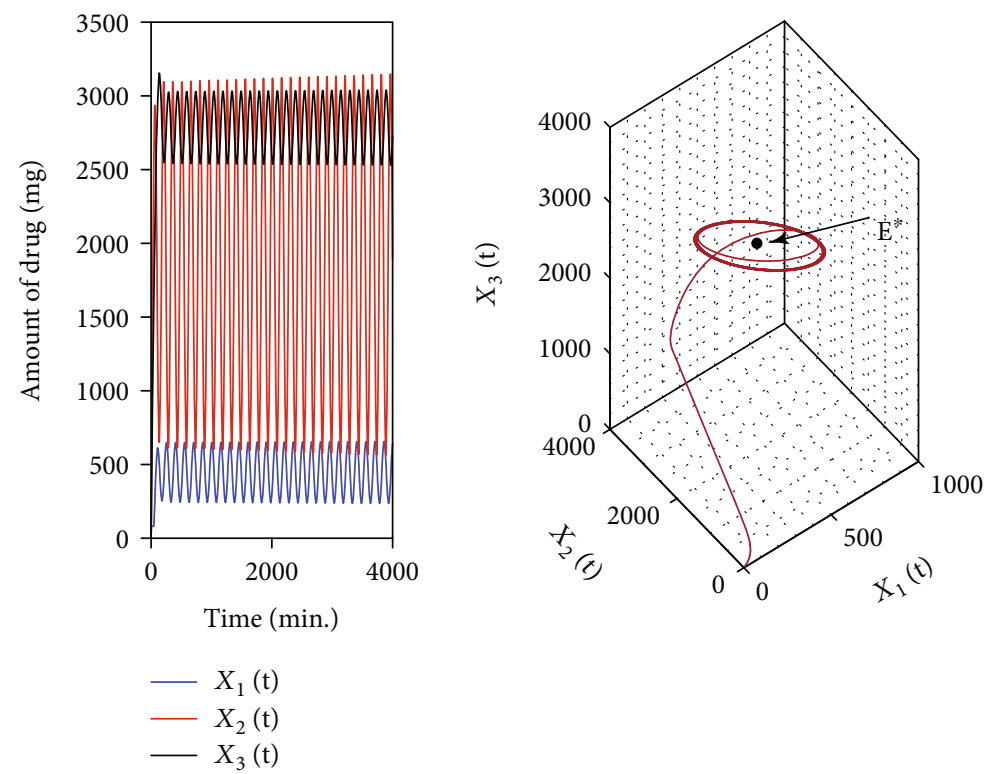

(b)

Figure 5: Numerical simulations for the local stability of trivial equilibrium $E^{*}$, displaying case 3, using parameter values in Table 1: (a) $\tau_{1}=\tau_{2}=25$, (b) $\tau_{1}=\tau_{2}=44.35$.

time delay is increased, as a results of the peaks, transient phase becomes longer. Finally, the whole pharmacokinetics process will be sensitive in its profile and duration as evident in Figures 6(a)-6(d).

The effects of variation in reabsorption rates $\left(k_{21}, k_{31}\right)$ and the drug's amount profiles at the compartments are depicted in Figures 7(a)-7(d). As can be seen, there are superimposition in all the initial phases of the profiles. However, as reabsorption rate from compartment 2 to 1 , where $k_{21}$ is varied $\left(k_{21}=0.0103,0.0206,0.1030\right.$ or $\left.k_{31}=0\right)$, the concentration of drug decreases in compartment 1 and increases in compartment 2 as shown in Figures 7(a) and 7(b), respectively. Similarly, when reabsorption rate from compartment 3 to 1 , where $k_{31}$ is varied $\left(k_{31}=0.00124\right.$, $0.00248,0.0124$ or $k_{21}=0$ ), the mass of drug decreases in compartment 1 while it increases in compartment 3 as evident from Figures 7(c) and 7(d), respectively.

The effects of elimination/excretion of drug from the three compartments are studied each by varying the corresponding rates. In Figure 8(a), the amount of drug profile in the central compartment is shown when the rate $k_{10}$ is varied $\left(k_{10}=0.0208,0.0416,0.208\right)$ and the other two rates of eliminations are fixed as $k_{20}=k_{30}=0$. In Figure 8(b) also, the amount profile in compartment 2 is displayed when the 


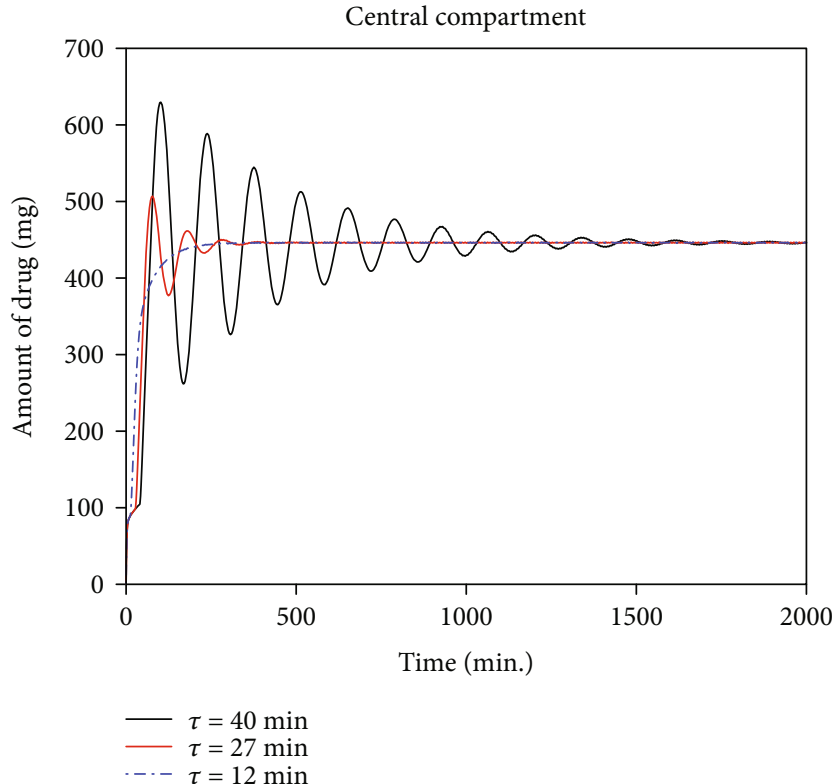

(a)

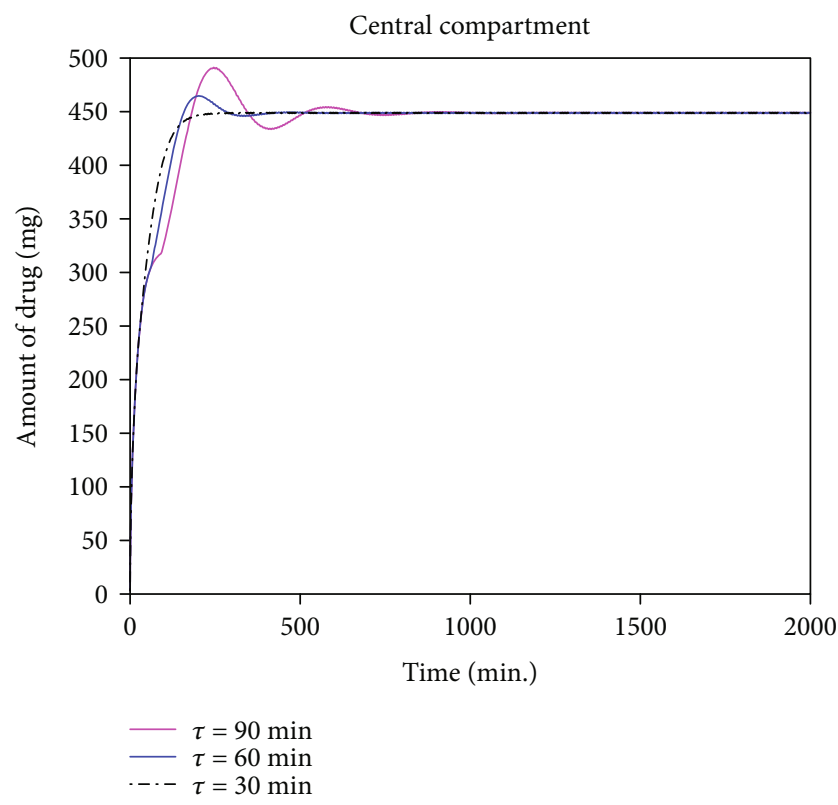

(c)

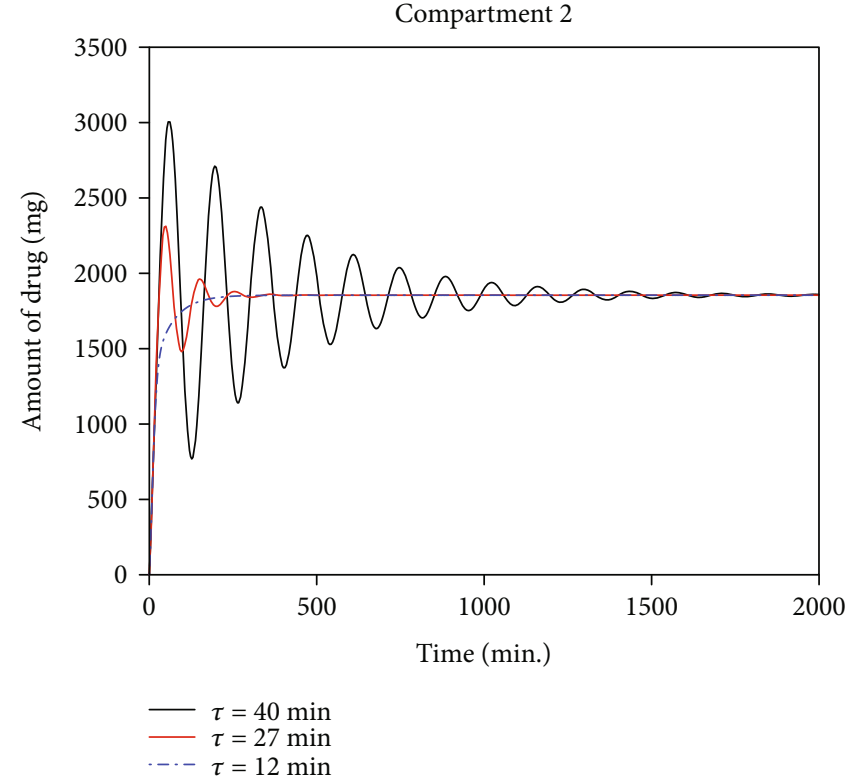

(b)

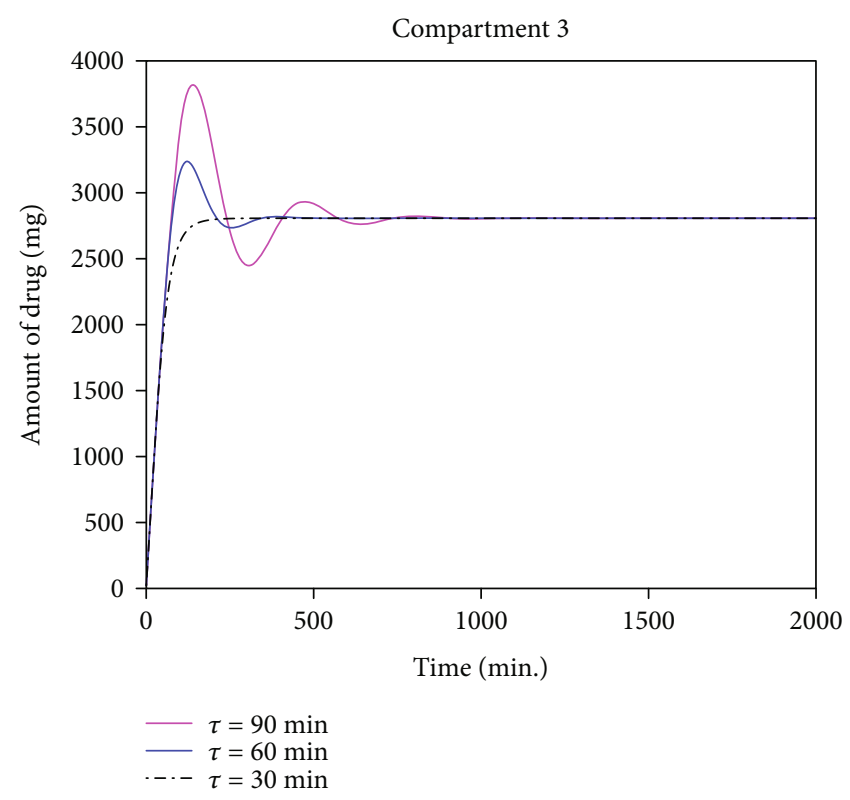

(d)

Figure 6: Time series for effects of time delay in reabsorption of drug from compartments 2 and 3 to the central compartment using parameter values in Table 1, except for (a) and (b) $\tau_{1}=12,27,40$ and $\tau_{2}=0.1$ (c) and (d) $\tau_{2}=30,60,90$ and $\tau_{1}=0.1$.

rate of elimination $k_{20}$ is varied $\left(k_{20}=0.000828,0.001656\right.$, 0.00828 ) while $k_{10}=k_{30}=0$. Similarly in Figure $8(\mathrm{c})$, the mass profile is shown when the elimination rate $k_{30}$ varies $\left(k_{30}=0.000784,0.001568,0.00784\right)$ or $k_{10}=k_{20}=0$. In all the three cases, it can be observed that as the rates are increased, the respective amounts in the compartments decreases significantly as can be seen in Figures 8(a)-8(c).

\section{Concluding Remarks}

In this article, a three-compartmental pharmacokinetics delay model is formulated and analyzed. The time delays represent the time lags in reabsorption of drug in the central compartment from two peripheral compartments. The model considers intravenous and oral administrations of drug to central and other two compartments, respectively. Furthermore, elimination of drug is considered in all the three compartments. Sensitivity analysis for effects of delays and parameter values obtained from published experimental data is performed numerically using MatLab dde23. Major findings of the research work are outlined below:

(1) Two equilibrium solutions are obtained. The trivial equilibrium of the model, when there is no drug 


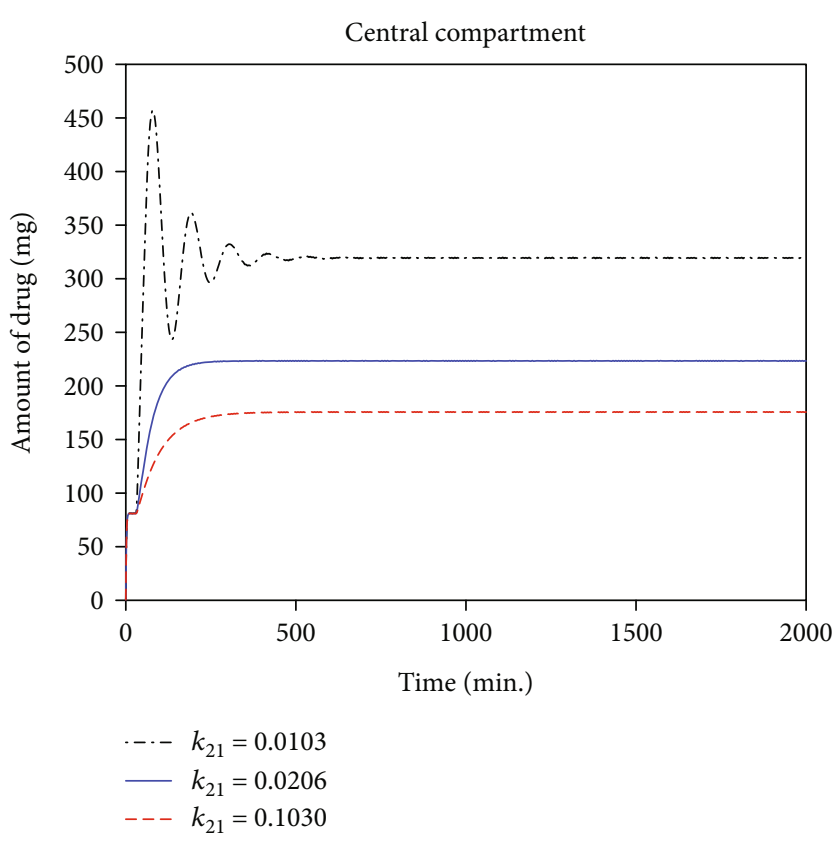

(a)

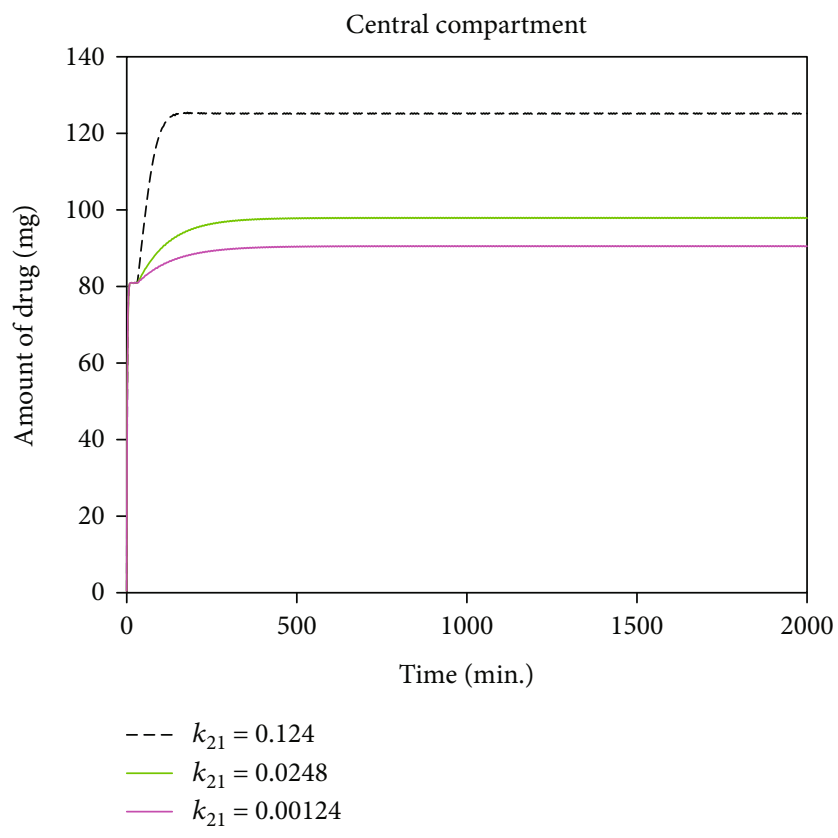

(c)

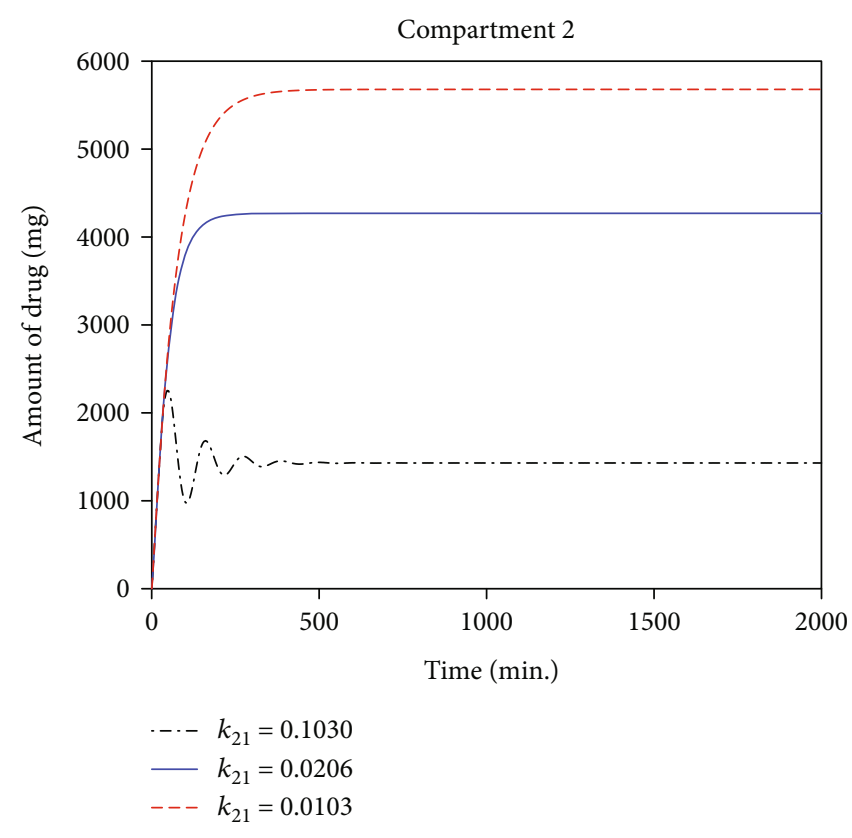

(b)

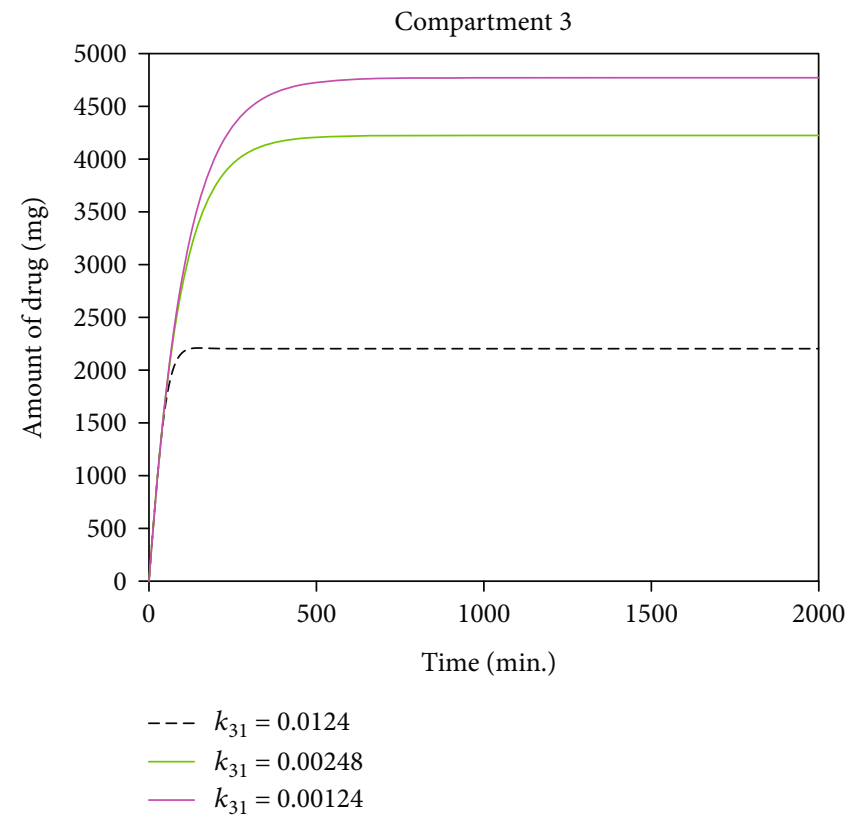

(d)

FIGURE 7: Time series for effect of reabsorption rates using parameter values in Table 1, except, $\tau_{1}=\tau_{2}=30$, in (a) and (b) $k_{21}=0.0103$, $0.0206,0.1030$ and $k_{31}=0$. In (c) and (d) $k_{31}=0.00124,0.00248,0.0124$ and $k_{21}=0$.

administered (intravenous or oral), is found to be locally asymptotically stable for all delay values. The biological meaning of this result is the initial amounts of drug in the compartments will vanish (approach zero) with time whenever there is no drug administered due to body metabolism. Peaks are also observed as reported in other models with delay. However, when there is either intravenous and/or oral doses, the nontrivial equilibrium emerged which is locally asymptotically stable for certain delay values. Thereafter, it becomes unstable and undergoes the Hopf bifurcation with family of periodic solutions bifurcating from the equilibrium. In this later case, the amount of drug can be determined within certain values of the delay as a result of delay effects in the model and thereafter, the amount fluctuates

(2) Effects of time lags in reabsorption of drugs by the central compartment from compartments 2 and 3 as delays are varied are evident. It is observed that as delays are increased, there are increases in number 


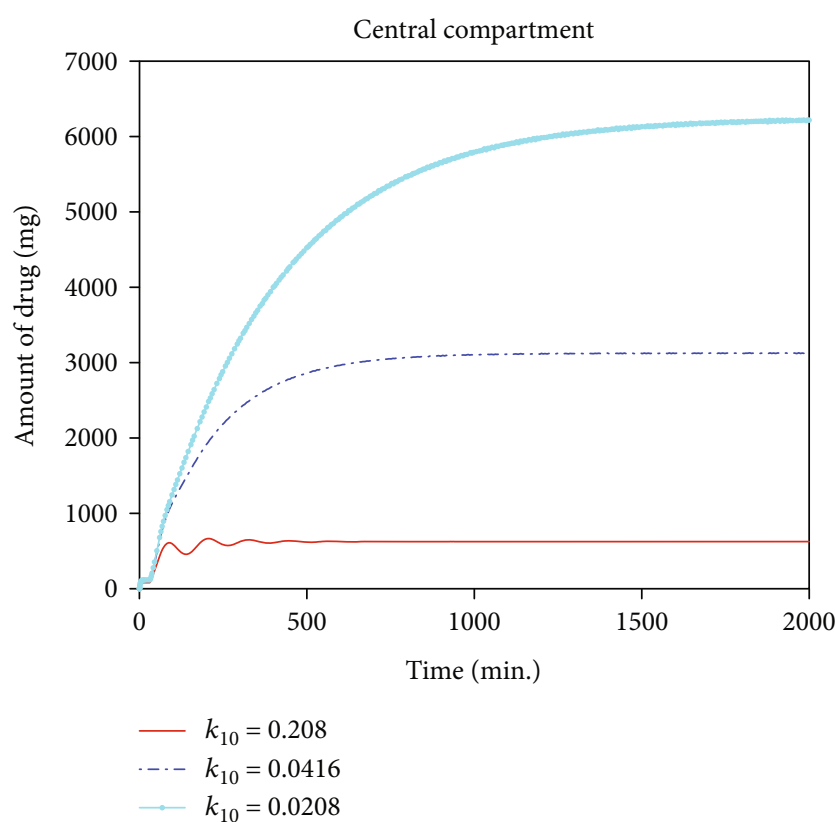

(a)

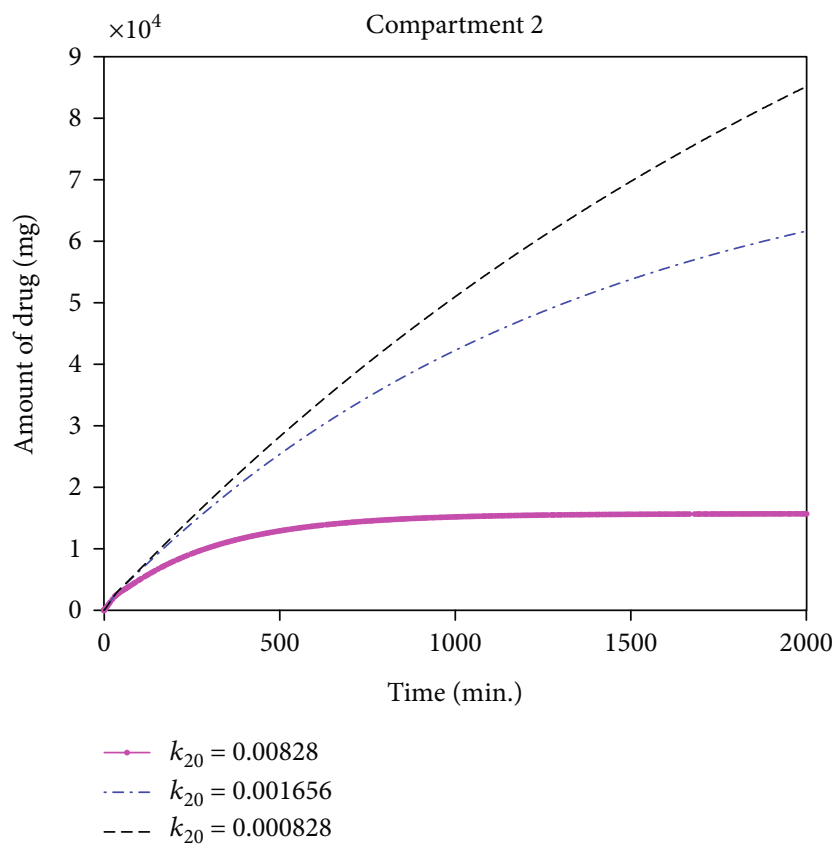

(b)

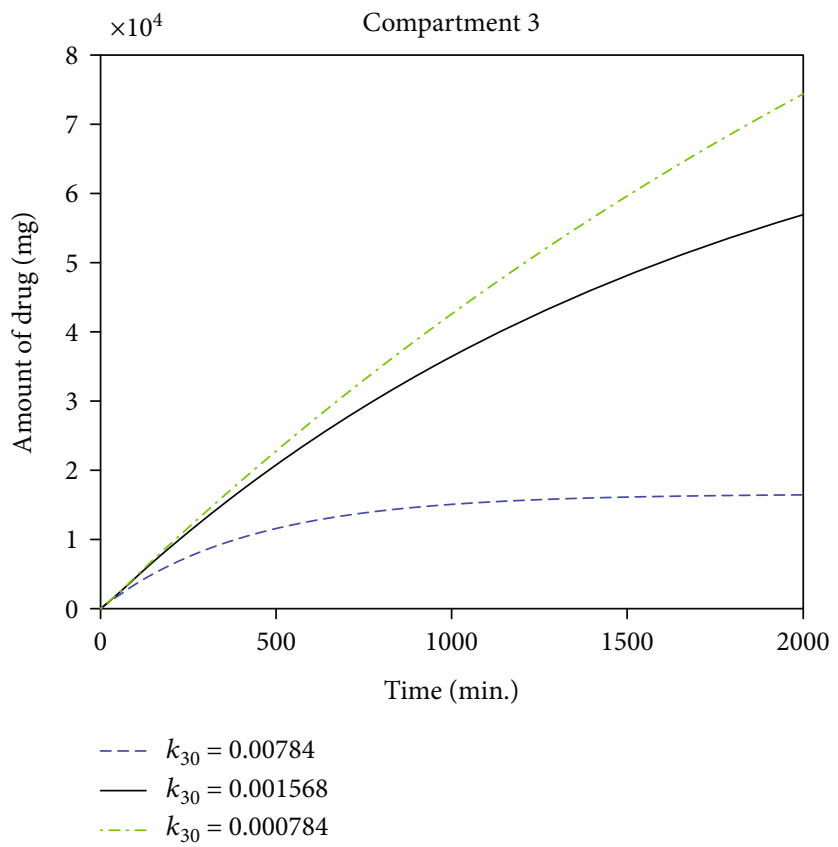

(c)

Figure 8: Time series for effects of elimination rates of drug using parameter values in Table 1, except $\tau_{1}=\tau_{2}=30$, in (a) $k_{10}=0.0208$, $0.0416,0.208$ and $k_{20}=k_{30}=0$, (b) $k_{20}=0.000828,0.001656,0.00828$ and $k_{10}=k_{30}=0$, (c) $k_{30}=0.000784,0.001568,0.00784$ and $k_{10}=k_{20}$ $=0$.

of rebounds or peaks in the time profiles of amounts in all the compartments. These coincides with the pharmacokinetics profiles in published delay models. Thus, the pharmacokinetics process is sensitive in its profile and duration in the presence of time delay

(3) When the reabsorption rates are increased, there are superimposition at the initial phases of the drug amount profiles; subsequently, the amount decreases in compartment 1 and increases in compartments 2 and 3 , respectively

(4) The effects of elimination/excretion of drug from the three compartments are studied each by varying the corresponding rates while other rates fixed. In all the compartments, it has been observed that as the rates are increased, the respective amount in the compartments decreases significantly. 


\section{Data Availability}

Data are found in published articles, as stated in the manuscript.

\section{Conflicts of Interest}

The authors hereby declare that there is no conflicts of interest whatsoever.

\section{Acknowledgments}

A. S. Hassan acknowledges with thanks the support of the Research Support Directorate, University of South Africa, for the Research Grant Fellowship number 409000. He is also grateful for the authority of Bayero University Kano, Nigeria, for the one-year research leave.

\section{References}

[1] T. Tozer and M. Rowland, Introduction to Pharmacokinetics and Pharmacodynamics: the Quantitative Basis of Drug Therapy, Lippincott Williams \& Wilkins, 2006.

[2] S. C. Turfus and B. J. Gurley, In: Pharmacognosy: Fundamentals, Applications and Strategies, Academic Press, 2017.

[3] N. H. G. Holford and L. B. Sheiner, "Kinetics of pharmacologic response," Pharmacology \& Therapeutics, vol. 16, no. 2, pp. 143-166, 1982.

[4] T. Teorell, "Kinetics of distribution of substances administered to the body, I: the extravascular modes of administration," Archives internationales de pharmacodynamie et de therapie, vol. 57, 1937.

[5] L. K. Paalzow, "Torsten Teorell, the Father of Pharmacokinetics," Upsala Journal of Medical Sciences, vol. 100, no. 1, pp. 4146, 1995.

[6] M. Gibaldi, Biopharmaceutics and Clinical Pharmacokinetics, Lea \& Febiger, Philadelphia, PA, USA, 4th edition, 1991.

[7] C. Labat, K. Mansour, M. F. Malmary, M. Terrissol, and J. Oustrin, "A variable reabsorption time-delay model for pharmacokinetics of drugs," Drug Metabolism and Pharmacokinetics, vol. 12, no. 2, pp. 129-133, 1987.

[8] J. L. Steimer, Y. Plusquellec, A. Guillaume, and J. F. Boisvieux, "A time-lag model for pharmacokinetics of drugs subject to enterohepatic circulation," Journal of pharmaceutical sciences, vol. 71, no. 3, pp. 297-302, 1982.

[9] Y. Plusquellec and L. Bousquet, "Time-delay for twocompartment models used for study of enterohepatic circulation of drugs," IEEE Transactions on Biomedical Engineering, vol. BME-31, no. 6, pp. 469-472, 1984.

[10] C. W. Sheppard, "The theory of the study of transfers within a multi-compartment system using isotopic tracers," Journal of Applied Physics, vol. 19, no. 1, pp. 70-76, 1948.

[11] I. Gyori, "Connections between compartmental systems with pipes and integro-differential equations," Mathematical Modelling, vol. 7, no. 9-12, pp. 1215-1238, 1986.

[12] S. Cascone, G. Lamberti, G. Titomanlio, O. Piazza, and U. Salerno, "Pharmacokinetics of Remifentanil: a threecompartmental modeling approach," Translational Medicine UniSa, vol. 7, no. 4, pp. 18-22, 2013.

[13] J. C. Péchère, M. M. Péchère, and R. Dugal, "Clinical pharmacokinetics of sisomicin: two-compartment model analysis of serum data after I.V. and I.M. administration," European Journal of Clinical Pharmacology, vol. 10, no. 3-4, pp. 251-256, 1976.

[14] E. B. Reeve and J. E. Roberts, "The kinetics of the distribution and breakdown of I131-albumin in the rabbit," The Journal of General Physiology, vol. 43, no. 2, pp. 415-444, 1959.

[15] L. Shargel and A. B. C. Yu, Applied Biopharmaceutics \& Pharmacokinetics, Mc Graw Hill Education, 2012.

[16] R. Bellman, "Topics in pharmacokinetics, II: Identification of time-lag processes," Mathematical Biosciences, vol. 11, no. 34, pp. 337-342, 1971.

[17] A. Mazanov, "Stability of multi-pool models with lags," Journal of Theoretical Biology, vol. 59, no. 2, pp. 429-442, 1976.

[18] T. Erneux, J. Javaloyes, M. Wolfrum, and S. Yanchuk, "Introduction to focus issue: time-delay dynamics," Chaos, vol. 27, no. 11, 2017.

[19] D. Huang, X. Zhang, Y. Guo, and H. Wang, "Analysis of an HIV infection model with treatments and delayed immune response," Applied Mathematical Modelling, vol. 40, no. 4, pp. 3081-3089, 2016.

[20] J. Liu, "Dynamical analysis of a delayed predator-prey system with modified Leslie-Gower and Beddington-DeAngelis functional response," Advances in Difference Equations, vol. 2014, no. 1,2014

[21] X. Wang, X. Q. Zhao, and J. Wang, "A cholera epidemic model in a spatiotemporally heterogeneous environment," Journal of Mathematical Analysis and Applications, vol. 468, no. 2, pp. 893-912, 2018.

[22] Y. Plusquellec and J. L. Steimer, "An analytical solution for a class of delay-differential equations in pharmacokinetic compartment modeling," Mathematical Biosciences, vol. 70, no. 1, pp. 39-56, 1984.

[23] O. F. Egbelowo, "Nonstandard finite difference approach for solving 3-compartment pharmacokinetic models," Biomedical Engineering, vol. 34, no. 9, 2018.

[24] J. K. Hale, Fnctional_Diff_Eqtns_Hale_1977.pdf, Springer, Berlin Heidelberg, NY, USA, 2nd edition, 1977.

[25] Y. Kuang, Delay Differential Equations with Applications in Population Dynamics, Academic Press, 1993.

[26] S. Ruan and J. Wei, "On the zeros of a third degree exponential polynomial with applications to a delayed model for the control of testosterone secretion," Journal of Mathematics Applied in Medicine and Biology, vol. 18, no. 1, pp. 41-52, 2001. 